\title{
Educar en Derechos Humanos: orientaciones del Derecho internacional e implementación en la educación superior
}

\author{
Educate in Human Rights: orientations of \\ international law and implementation in higher \\ education
}

\author{
Vicente Bellver Capella \\ Catedrático de Filosofía del Derecho y Política \\ Departamento de Filosofía del Derecho y Política. \\ Facultad de Derecho. \\ Universitat de València. \\ E-mail: vicente.bellver@uv.es
}

\begin{abstract}
Resumen: La Declaración Universal de Derechos Humanos considera que uno de los objetivos de la educación es "el fortalecimiento del respeto a los derechos humanos". Tras la Conferencia de Viena de Derechos Humanos, Naciones Unidas y otras agencias intergubernamentales de alcance regional vienen impulsando la Educación en Derechos Humanos (EDH). En este trabajo se hace un repaso histórico de las principales iniciativas a nivel internacional dedicados a la EDH. Se presta especial atención a las políticas y normas aprobadas por Naciones Unidas y el Consejo de Europa. Finalmente se reflexiona sobre la importancia y dificultades poner en marcha la EDH en el ámbito de la educación superior.
\end{abstract}

Palabras clave: Derechos humanos; Educación; Educación en derechos humanos; Educación cívica; Universidad.

Abstract: According to the Universal Declaration of Human Rights, one of the main goals of education is "the strengthening of respect for human rights and fundamental freedoms" (art.26). After the World Conference on Human Rights (Vienna, 1993), United Nations and other intergovernmental regional agencies have been boosting Human Rights Education (HRE). This paper presents the most relevant international initiatives in the field of HRE. Particular attention is devoted to the documents delivered by United Nations and the Council of Europe on this topic. Finally, I reflect on the importance and difficulties to develop HRE at the University.

Keywords: Human rights; Education; Human Rights Education; Civic Education; University. 


\title{
Educar en Derechos Humanos: orientaciones del Derecho internacional e implementación en la educación superior.
}

\author{
Educate in Human Rights: orientations of international law and implementation \\ in higher education.
}

En este trabajo hago un repaso histórico de las iniciativas que las organizaciones intergubernamentales han llevado a cabo en el campo de la educación en los derechos humanos (EDH) desde mediados del pasado siglo hasta la actualidad. En primer lugar, me ocupo de la labor emprendida por Naciones Unidas. A continuación, hago referencia a las iniciativas del Consejo de Europa en esta materia. Concluyo con una reflexión sobre la urgencia de llevar la EDH a la enseñanza superior, las dificultades que se plantean para conseguirlo, y algunas propuestas que el gobierno español debería emprender para cumplir con el marco internacional presentado y con el que España está comprometida.

\section{Las Naciones Unidas y la educación en derechos humanos (EDH).}

Tanto la educación y como los derechos humanos son imprescindibles para el desarrollo de la vida personal y social. Además, están íntimamente relacionados, de modo que es imposible que los derechos humanos se respeten si las personas no son educadas para su respeto y defensa; y no se alcanzará una verdadera educación de las personas si no se garantizan todos sus derechos, incluido obviamente el derecho a la educación, y a una educación conforme a los derechos humanos. Esta íntima relación fue puesta de manifiesto en la misma Declaración Universal de Derechos Humanos y, desde entonces, se ha reconocido siempre la necesidad de considerarlos conjuntamente.

\section{1.- Las bases de la educación en derechos humanos: la Declaración Universal de} Derechos humanos y El Pacto Internacional de Derechos Económicos, Sociales y Culturales.

Cuando la Asamblea General de Naciones Unidas aprobó la Declaración Universal de Derechos Humanos (DUDH) en 1948 no pretendía que ese breve documento, que fue resultado de un difícil consenso, y que ni siquiera contó con la aprobación de todos los estados existentes en el mundo en aquel momento, lograra la implantación real de los derechos humanos en todo el mundo. En el Preámbulo se señala un objetivo que es, a su vez, más realista y más ambicioso: "La Asamblea General proclama la presente 
Declaración Universal de Derechos Humanos como ideal común por el que todos los pueblos y naciones deben esforzarse, a fin de que tanto los individuos como las instituciones, inspirándose constantemente en ella, promuevan mediante la enseñanza y la educación, el respeto a estos derechos y libertades". Siendo un texto jurídico, no fía su éxito a las garantías jurídicas que los estados aprueben. Más bien apuesta por la educación como instrumento para conseguir el respeto universal de los derechos. En coherencia con ello, cuando la DUDH proclama el derecho a la educación en su artículo 26.2 dice expresamente: "La educación tendrá por objeto el pleno desarrollo de la personalidad humana y el fortalecimiento del respeto a los derechos humanos y a las libertades fundamentales; favorecerá la comprensión, la tolerancia y la amistad entre todas las naciones y todos los grupos étnicos o religiosos; y promoverá el desarrollo de las actividades de las Naciones Unidas para el mantenimiento de la paz".

El artículo 1 de la DUDH dice: “Todos los seres humanos nacen libres e iguales en dignidad y derechos y, dotados como están de razón y conciencia, deben comportarse fraternalmente los unos con los otros". El artículo 29, por su parte, afirma que "toda persona tiene deberes respecto a la comunidad, puesto que sólo en ella puede desarrollar libre y plenamente su personalidad”. El primero de estos artículos asegura que el deber de comportamiento fraternal entre los seres humanos es la consecuencia debida de la igual dignidad de cada uno de ellos. El segundo afirma que los deberes hacia los demás no son un peaje que tenemos que pagar para poder hacer a cambio lo que verdaderamente queremos, sino la condición para la plena realización de nuestra personalidad. Por tanto, debemos portarnos fraternalmente con los demás seres humanos porque todos son igualmente dignos. Y cuando lo hacemos, lejos de sufrir una autolimitación, contribuimos a nuestra plena realización personal. Si ponemos en relación estos dos artículos con el 26 podemos concluir que la educación tiene entre sus fines el fortalecimiento del respeto de los derechos humanos y el cumplimiento de los deberes respecto a la comunidad; y que su consecución trae consigo el pleno desarrollo de la personalidad de los individuos.

Ahora bien, la educación es imposible si no se garantizan los derechos humanos (entre ellos, obviamente, el derecho a la educación) y si la acción educativa no se lleva a cabo desde el escrupuloso respeto a los derechos humanos (Bellver, 2013: 269-287). ¿Cómo se puede educar a alguien si la escuela más próxima está a un centenar de kilómetros, si vive en un país en el que las libertades políticas están recortadas o si tiene que consumir tantas energías para cubrir sus necesidades básicas que ya no le quedan fuerzas para 
dedicarse a su educación? ¿O cómo se puede pretender hablar de educación si, en lugar de promover la libertad del individuo, la actividad presuntamente educativa trata más bien de manipularlo?

A la vista de lo que venimos diciendo, podemos concluir que la educación tiene como uno de sus fines esenciales fomentar el respeto de los derechos humanos (así lo reconoce el art. 26.2 de la DUDH); y que la garantía efectiva de los derechos humanos es condición fundamental para que la educación pueda ejercerse y cumplir su fin. Derechos humanos y educación se imbrican de tal manera que generan una espiral que, como todas, tiene un sentido tanto ascendente como descendente. Cuando es ascendente, la educación conduce a reforzar el respeto de los derechos humanos, lo que redunda en un fortalecimiento de la calidad educativa y así sucesivamente. Cuando es descendente, la violación de los derechos humanos dificulta la acción educativa, lo que debilita el respeto a los derechos humanos y fomenta aún más su violación, haciendo prácticamente imposible la educación.

En continuidad con lo establecido en la DUDH, el art. 13 del Pacto Internacional de Derechos Económicos, Sociales y Culturales (PIDESC), aprobado en 1966 y en vigor desde 1977, afirma: "1. Los Estados Partes en el presente Pacto reconocen el derecho de toda persona a la educación. Convienen en que la educación debe orientarse hacia el pleno desarrollo de la personalidad humana y del sentido de su dignidad, y debe fortalecer el respeto por los derechos humanos y las libertades fundamentales. Convienen asimismo en que la educación debe capacitar a todas las personas para participar efectivamente en una sociedad libre, favorecer la comprensión, la tolerancia y la amistad entre todas las naciones y entre todos los grupos raciales, étnicos o religiosos, y promover las actividades de las Naciones Unidas en pro del mantenimiento de la paz". Además de reproducir lo dispuesto en el art. 26.2 de la DUDH, el art. 13.1 del PIDESC añade que la educación debe orientarse al desarrollo del sentido de la dignidad de la persona. Dentro de la ambiguiedad de los términos, resulta plausible interpretar que la educación no sólo debe hacer hincapié en el desarrollo de la libertad individual sino también en la conciencia del valor de toda persona por el mero hecho de serlo. Aunque este añadido no siempre ha sido tenido en cuenta en los documentos posteriores de Naciones Unidas que se han ocupado del derecho a la educación (por ejemplo, no se recoge en el art. 29.1 de la Convención sobre los Derechos del Niño, que trata de los 
objetivos de la educación ${ }^{1}$ ), considero que refuerza la relación que cabe establecer entre los arts. 1 y 29 con el art. 26, todos de la DUDH.

\section{2.- La educación para los derechos humanos en el marco de Naciones Unidas. \\ a.- La Recomendación de la UNESCO de 1974.}

La DUDH afirmó que la educación ha de perseguir el respeto de los derechos humanos.

Pero será a partir de los años setenta cuando Naciones Unidas emprenda una ambiciosa estrategia, que ha ido creciendo en intensidad, dirigida a que realmente la educación en todo el mundo incorpore entre sus objetivos el respeto de los derechos humanos.

En 1974 la Conferencia General de la Organización de las Naciones Unidas para la Educación, la Ciencia y la Cultura (UNESCO) aprobó la "Recomendación sobre la educación para la comprensión, la cooperación y la paz internacionales y la educación relativa a los derechos humanos y las libertades fundamentales" ${ }^{2}$. Se recomienda a los Estados Miembros que adopten medidas para dar efecto a los principios rectores de las políticas educativas recogidos en la Recomendación ${ }^{3}$, que harán posible la consecución de ese objetivo.

Todo el texto de la Recomendación es tan claro y concreto como los principios rectores que consagra y, a pesar del tiempo transcurrido desde su aprobación, no ha quedado obsoleta ni olvidada por la UNESCO. De hecho, el Comité de Convenciones y Recomendaciones del Consejo Ejecutivo de la UNESCO se ha venido preocupando de examinar periódicamente el nivel de aplicación de esta Recomendación por parte de los estados miembros. Lo ha hecho en seis ocasiones, la última de las cuales se llevó a cabo

\footnotetext{
1 “Art. 29.1. Los Estados partes convienen en que la educación del niño deberá estar encaminada a: a. Desarrollar la personalidad, las aptitudes y la capacidad mental y física del niño hasta el máximo de sus posibilidades;

b. Inculcar al niño el respeto de los Derechos Humanos y las Libertades Fundamentales y de los principios consagrados en la carta de las Naciones Unidas;

c. Inculcar al niño el respeto de sus padres, de su propia identidad cultural, de su idioma y sus valores, de los valores nacionales del país en que vive, del país de que sea originario y de las civilizaciones distintas de la suya;

d. Preparar al niño para asumir una vida responsable en una sociedad libre, con espíritu de comprensión, paz, tolerancia, igualdad de los sexos y amistad entre todos los pueblos, grupos étnicos, nacionales y religiosos y personas de origen indígena;

e. Inculcar al niño el respeto del medio ambiente natural".

2 http://www.unesco.org/education/nfsunesco/pdf/PEACE_S.PDF (acceso el 22 de febrero de 2018).

3 "a) una dimensión internacional y una perspectiva global de la educación en todos sus niveles y en todas sus formas; b) la comprensión y el respeto de todos los pueblos, sus culturas, civilizaciones, valores y modos de vida, incluidas las culturas étnicas tanto nacionales como las de otras naciones; c) el reconocimiento de la creciente interdependencia mundial de los pueblos y las naciones; d) la capacidad de comunicarse con los demás; e) el conocimiento no sólo de los derechos, sino de los deberes que tienen las personas, los grupos sociales y las naciones para con los demás; f) la comprensión de la necesidad de la solidaridad y la cooperación internacionales; g) la disposición por parte de cada uno de participar en la solución de los problemas de su comunidad, de su país y del mundo entero".
} 
en 2017 (UNESCO, 2017) y dio lugar a la publicación, como en las ocasiones anteriores, de un informe en el que se reseñaban las dificultades para secundar esta Recomendación.

Aunque no ha tenido el impacto de la Recomendación, conviene hacer referencia a la Declaración y Plan de Acción Integrado sobre la Educación para la Paz, los Derechos Humanos y la Democracia, aprobada en la $44^{\text {a }}$ Conferencia Internacional de Educación en 1994, y ratificada por la UNESCO al año siguiente. En ella se dice que "las instituciones de enseñanza superior pueden contribuir de múltiples maneras a la educación para la paz, los derechos humanos y la democracia. En este sentido, debería considerarse la posibilidad de introducir, en los programas de estudios, conocimientos, valores y aptitudes referentes a la paz, los derechos humanos, la justicia, la práctica de la democracia, la ética profesional, el civismo y la responsabilidad social. Las instituciones de enseñanza de este nivel también deberían velar por que los estudiantes tomen conciencia de la interdependencia de los Estados en una sociedad cada vez más mundializada" (UNESCO, 1995).

\section{b.- La $2^{a}$ Conferencia Mundial de Derechos Humanos (Viena, 1993).}

La Declaración y Programa de Acción de Viena, aprobados por la Conferencia Mundial de Derechos Humanos en 1993 es el principal fruto de aquella reunión, la segunda sobre derechos humanos celebrada hasta el momento en el marco de Naciones Unidas. Este documento se refiere a la cuestión de la educación en derechos humanos en cada una de las dos grandes partes en que está dividido. En el n. 33 de la parte I se dice: "La Conferencia Mundial de Derechos Humanos reitera el deber de los Estados, explicitado en la Declaración Universal de Derechos Humanos, en el Pacto Internacional de Derechos Económicos, Sociales y Culturales y en otros instrumentos internacionales de derechos humanos, de encauzar la educación de manera que se fortalezca el respeto de los derechos humanos y las libertades fundamentales. La Conferencia destaca la importancia de incorporar la cuestión de los derechos humanos en los programas de educación y pide a los Estados que procedan en consecuencia. La educación debe fomentar la comprensión, la tolerancia, la paz y las relaciones de amistad entre las naciones y entre los grupos raciales o religiosos y apoyar el desarrollo de las actividades de las Naciones Unidas encaminadas al logro de esos objetivos. En consecuencia, la educación en materia de derechos humanos y la difusión de información adecuada, sea de carácter teórico o práctico, desempeñan un papel 
importante en la promoción y el respeto de los derechos humanos de todas las personas sin distinción alguna por motivos de raza, sexo, idioma o religión y debe integrarse en las políticas educativas en los planos nacional e internacional”. En este párrafo no se hace más que reiterar lo que venía proponiendo Naciones Unidas desde la DUDH.

La parte II de la Declaración y Programa de Acción dedica el apartado D a la "Educación en materia de derechos humanos". En los cinco parágrafos dedicados al asunto (parr. 78-82) destacan las siguientes manifestaciones:

- “79. (...) La Conferencia Mundial de Derechos Humanos pide a todos los Estados e instituciones que incluyan los derechos humanos, el derecho humanitario, la democracia y el imperio de la ley como temas de los programas de estudio de todas las instituciones de enseñanza académica y no académica". Se trata de una petición a los Estados para que, entre otras cosas, incorporen en los planes de estudio la formación en derechos humanos. Pero va más allá, al pedir que la educación incorpore la democracia. Es lógico que así fuera pues no parece posible fortalecer el respeto de los derechos humanos si no se fortalece igualmente la democracia ${ }^{4}$.

- "80. La educación en materia de derechos humanos debe abarcar la paz, la democracia, el desarrollo y la justicia social, tal como se dispone en los instrumentos internacionales y regionales de derechos humanos...". La Conferencia considera que la formación en derechos humanos se ha de basar en los instrumentos internacionales dedicados a ellos. Este planteamiento ofrece una ventaja y un inconveniente. Al ceñir la educación en derechos humanos a lo dispuesto en los instrumentos normativos aprobados por la comunidad internacional es más sencillo recabar un consenso social amplio hacia este tipo de iniciativa, habida cuenta de que dichos textos ya han sido aprobados por la mayoría de los estados y suelen estar redactados en términos suficientemente indeterminados como para que las mayorías sociales puedan sentirse identificadas con ellos. El inconveniente está en el riesgo de reducir la educación en derechos humanos a lo establecido en esos documentos. Si la educación en derechos humanos consiste en dar a conocer lo dispuesto por los organismos internacionales en esta materia, resulta difícil evitar una visión excesivamente positivista de los derechos humanos. Los derechos humanos son ciertamente lo que resulta de las normas internacionales, pero no sólo. Son también un instrumento de lucha por la dignidad; son

\footnotetext{
${ }^{4}$ Para aproximarse a las complejidades de la educación para la democracia y conocer la propuesta que procede de los teóricos de la democracia deliberativa, ver: Guttman, A. (2001).
} 
un lugar de encuentro y de debate; son el resultado siempre variable de interpretar un sinnúmero de textos sumamente ambiguos; etc.

- "82. (...) Los programas de servicios de asesoramiento y asistencia técnica del sistema de los Estados relacionados con las Naciones Unidas deben poder atender inmediatamente a las solicitudes de actividades educacionales y de formación en la esfera de los derechos humanos y con la educación especial en lo que respecta a las normas contenidas en los instrumentos internacionales de derechos humanos y en el derecho humanitario así como a su aplicación, destinada a grupos especiales, como fuerzas militares, fuerzas del orden, policía y personal de salud". Cuando se habla de educación en derechos humanos se tiende a pensar en los niños y los jóvenes, y es lógico que sea así. Pero, como bien se señala en este punto, se debe prestar una atención especial a aquellos colectivos que ostentan una posición de poder, bien porque están legitimados para el uso de la fuerza física (fuerzas armadas y cuerpos de seguridad del Estado) o bien porque tratan con las personas en situaciones e especial vulnerabilidad (los profesionales de la sanidad o de la educación).

- "82. Debe considerarse la posibilidad de proclamar un Decenio de las Naciones Unidas para la educación en materia de derechos humanos a fin de promover, alentar y orientar estas actividades educacionales". Esta propuesta fue atendida por la Asamblea General de Naciones Unidas que, en su resolución 49/184 del 23 de diciembre de 1994, proclamó que el período de diez años a partir del $1^{\text {o }}$ de enero de 1995, sería el Decenio de las Naciones Unidas para la educación en la esfera de los derechos humanos.

c.- El Decenio de las Naciones Unidas para la educación en la esfera de los derechos humanos y Programa Mundial para la Educación en Derechos Humanos.

Este Decenio ${ }^{5}$ se articuló en torno a un plan, elaborado por el Secretario General de Naciones Unidas, cuyos objetivos fueron cinco:

“a) La evaluación de las necesidades y la formulación de estrategias eficaces para el fomento de la educación en la esfera de los derechos humanos en todos los niveles escolares, en la capacitación profesional y en la enseñanza académica y no académica;

b) La creación y fortalecimiento de programas y capacidades para la educación en la esfera de los derechos humanos en los planos internacional, regional, nacional y local;

c) El desarrollo coordinado de los materiales didácticos para la enseñanza de los derechos humanos;

\footnotetext{
${ }^{5}$ Un riguroso relato de la génesis de este Decenio y del posterior Programa Mundial para la Educación en Derechos Humanos: Acebal Monfort, L. (2006: 41-66).
} 
d) El fortalecimiento de la función y la capacidad de los medios de difusión en el fomento de la educación en la esfera de los derechos humanos;

e) La difusión mundial de la Declaración Universal de Derechos Humanos en el mayor número posible de idiomas y en otras formas apropiadas para los diversos niveles de instrucción y para las personas discapacitadas" (Naciones Unidas, 1994).

Se trata de unos objetivos de muy desigual alcance. Así como los objetivos c) y e) son más o menos asequibles, los otros tres son tareas inabarcables, como la evaluación de los resultados del Plan pusieron de manifiesto ${ }^{6}$. En todo caso, se refieren a tres áreas en las que es fundamental actuar para lograr una verdadera educación en derechos humanos. En primer lugar, es necesario incorporar esa educación a todos los niveles de la enseñanza formal e informal. En segundo, todas las administraciones públicas deben comprometerse con la educación en derechos humanos en su esfera propia de competencias. Por último, los medios de comunicación tienen que implicarse decididamente pues ellos condicionan la educación informal que reciben los ciudadanos y que tanto repercute en su forma de pensar y actuar. Este quizá sea uno de los aspectos más críticos pues, si bien el éxito de la educación en derechos humanos es imposible sin la complicidad de los medios, solo se cuenta con el magro recurso de la persuasión para lograr su implicación.

El plan preveía la elaboración por parte de los Estados de planes nacionales sobre educación en derechos humanos, así como evaluaciones periódicas sobre su nivel de cumplimiento. A la vista de los modestos objetivos alcanzados a lo largo del Decenio, y de la necesidad de plazos más amplios para incorporar determinados cambios en la educación, la consecuencia principal del Informe de evaluación fue proponer un segundo Decenio que, finalmente, dio pie al Programa Mundial para la Educación en Derechos Humanos (Comisión de Derechos Humanos, 2004b) aprobado mediante Resolución de la Asamblea General de Naciones Unidas (Naciones Unidas, 2005).

\footnotetext{
${ }^{6}$ Llama la atención el hecho de que sólo 28 Estados respondieran el cuestionario enviado por el Alto Comisionado para los Derechos Humanos de Naciones Unidas para recabar la información necesaria para elaborar el Informe de evaluación del Decenio. En ese Informe se destaca, como principal conclusión, que el objetivo para la educación en derechos humanos no se ha alcanzado suficientemente y que requeriría al menos de otro decenio para lograrlo (Comisión de Derechos Humanos, 2004a). Es interesante hacer notar que dicho informe recoge -como uno de los fallos del Decenio en opinión de alguno de los Estados- el hecho de que "algunos de los asuntos no recibieron una cobertura adecuada durante el Decenio en cuanto al contenido de las actividades de educación en la esfera de los derechos humanos, en particular, los derechos económicos, sociales y culturales o que éstos no se contemplen en la medida en que se debiera como parte del conjunto indivisible de los derechos humanos; la cuestión de las responsabilidades conexas; el medio ambiente; y los derechos humanos de la mujer" (n. 24). Aunque no es el momento de abundar en este tema, pone de manifiesto la enorme dificultad que plantea la educación en derechos humanos: qué derechos abarca, qué contenido tiene cada uno; qué relación existe entre ellos; qué importancia específica debe darse a determinados derechos humanos en cada momento; etc.
} 
Los objetivos del Programa son más concretos y ambiciosos (Acebal Monfort, 2006: 43) que los aprobados para el Decenio: “a) Contribuir a forjar una cultura de derechos humanos; b) Promover el entendimiento común, sobre la base de los instrumentos internacionales, de los principios y metodologías básicos para la educación en derechos humanos; c) Asegurar que la educación en derechos humanos reciba la debida atención en los planos nacional, regional e internacional; d) Proporcionar un marco colectivo común para la adopción de medidas por todos los agentes pertinentes; e) Aumentar la cooperación y la asociación en todos los niveles; f) Examinar, evaluar y apoyar los programas de educación en derechos humanos existentes, poner de relieve las prácticas satisfactorias y proporcionar incentivos para continuarlas o ampliarlas y para crear prácticas nuevas; g) Promover la aplicación de la Declaración de las Naciones Unidas sobre educación y formación en materia de derechos humanos".

Este Programa, a diferencia del Decenio, se estructura en etapas consecutivas dirigidas a intensificar las actividades nacionales de educación en derechos humanos en sectores determinados. En estos momentos se está desarrollando la tercera etapa. La primera (2005-2009) se centró en los sistemas de enseñanza de primaria y secundaria. En un principio, esta primera etapa del Programa Mundial se puso en marcha por tres años (hasta 2007). Posteriormente, el Consejo de Derechos Humanos decidió prolongarla dos años, hasta fines de 2009. La segunda etapa (2010-2014) puso el foco sobre la educación en derechos humanos para la enseñanza superior y los programas de capacitación para maestros y educadores, funcionarios públicos, fuerzas del orden y personal militar ${ }^{7}$. La primera etapa se cerró con la elaboración de un informe. En este caso 76 Estados enviaron informaciones sobre los resultados alcanzados, un número sensiblemente superior a los que respondieron cuando se elaboró el informe de evaluación del Decenio. No obstante, se sigue constatando en el informe que los pasos que se van dando son mucho más lentos de lo deseable (Naciones Unidas, 2010).

Al igual que en la primera etapa del Programa, también la segunda contó con un Plan de Acción. Pero resulta curioso advertir que, si bien en ambos planes se hace mención a los principios rectores que deben inspirar las actividades sobre educación en derechos humanos, la relación de principios no es del todo coincidente. No es el momento de

\footnotetext{
${ }^{7}$ El Consejo de Derechos Humanos: "decide centrar la segunda etapa del Programa Mundial para la educación en derechos humanos en la enseñanza superior y en los programas de capacitación sobre derechos humanos para maestros y educadores, funcionarios públicos, fuerzas del orden y personal militar a todos los niveles" (Consejo de Derechos Humanos, 2009).
} 
analizar las diferencias del segundo $\operatorname{plan}^{8}$ con respecto al primero (Alto Comisionado de las Naciones Unidas para los Derechos Humanos, 2005). Sea como fuere, no creo que estos cambios -que se producen nada más y nada menos que en los principios rectores de la educación en los derechos humanos en el corto plazo de cinco años- contribuyan a dar seriedad, solidez y seguridad al Programa. ¿Quién nos puede garantizar que los principios rectores de una ulterior etapa del Programa no contengan discrepancias sustanciales con respecto a los consagrados en etapas anteriores? Y entonces, ¿por qué tomarse tan en serio los principios proclamados ahora si en un futuro no lejano podrían cambiar? Igual que se hizo en la primera etapa, también la segunda concluyó con un informe de evaluación, que solo contó con las informaciones aportadas por 36 estados. En las conclusiones de ese informe se destaca la importancia de que la tercera etapa del programa sirva para consolidar los objetivos de las dos primeras, y se centre particularmente en la enseñanza superior y la formación de maestros y educadores, funcionarios públicos, agentes del orden y personal militar (Alto Comisionado de las Naciones Unidas para los Derechos Humanos, 2015).

En este momento está concluyendo la tercera etapa del Programa Mundial para la Educación en Derechos Humanos (2015-2019) que, como ya se anunciaba en el informe de evaluación mencionado, tiene como objetivo principal consolidar los que se propusieron en las dos etapas anteriores y promover la formación en derechos humanos de los profesionales de los medios de comunicación y los periodistas (Alto Comisionado de las Naciones Unidas para los Derechos Humanos, 2014).

\section{d.- La Declaración de Naciones Unidas sobre la educación y formación en materia de} derechos humanos y los ODS.

Más allá de los logros alcanzados hasta el momento en materia de educación de derechos humanos gracias a las dos grandes iniciativas promovidas por Naciones Unidas desde 1995 (y que tienen su origen en la propuesta de la Conferencia Mundial de Derechos Humanos de 1993), el fruto normativo más relevante a escala universal es, sin duda, la aprobación en diciembre de 2011, mediante Resolución de la Asamblea General de Naciones Unidas, de la Declaración sobre la educación y formación en materia de derechos humanos (Asamblea General de Naciones Unidas, 2012). Se trata de un texto que sintetiza lo que, de forma dispersa, se había venido manifestando en un gran número de instrumentos jurídicos aprobados por Naciones Unidas a lo largo de los

\footnotetext{
${ }^{8}$ El único cambio verdaderamente relevante consistió en incluir la referencia a la discriminación por razón de la orientación sexual (Consejo de Derechos Humanos, 2010).
} 
últimos quince años. Pero no se queda aquí, sino que aporta novedades relevantes como las siguientes:

- El art. 1.1 proclama lo que podría entenderse como un tímido reconocimiento del derecho humano a la educación en derechos humanos. Lo hace en los siguientes términos: "Toda persona tiene derecho a poseer, buscar y recibir información sobre todos los derechos humanos y las libertades fundamentales y debe tener acceso a la educación y la formación en materia de derechos humanos" ${ }^{\prime 9}$. No sólo se reconoce la libertad de buscar información, sino el deber del Estado de garantizar a todas las personas educación en materia de derechos humanos.

- El art. 2.2 ordena, como se había hecho hasta ahora en un documento de estas características, las categorías de acción implicadas en la expresión educación en derechos humanos: "La educación y la formación en materia de derechos humanos engloban:

a) La educación sobre los derechos humanos, que incluye facilitar el conocimiento y la comprensión de las normas y principios de derechos humanos, los valores que los sostienen y los mecanismos que los protegen;

b) La educación por medio de los derechos humanos, que incluye aprender y enseñar respetando los derechos de los educadores y los educandos;

c) La educación para los derechos humanos, que incluye facultar a las personas para que disfruten de sus derechos y los ejerzan, y respeten y defiendan los de los demás".

- La Declaración destaca en su art. 4 la importancia de las tecnologías de la información y la comunicación, los medios de comunicación en general, y las artes en la formación en derechos humanos: “1. La educación y la formación en materia de derechos humanos deben aprovechar y utilizar las nuevas tecnologías de la información y las comunicaciones, así como los medios de comunicación, para promover todos los derechos humanos y las libertades fundamentales. 2. Deben alentarse las artes como medio de formación y sensibilización en la esfera de los derechos humanos". Es una pena que, junto a las artes, no se incluyera una referencia al conjunto de las humanidades como instrumento privilegiado para educar en los derechos humanos ${ }^{10}$.

\footnotetext{
${ }^{9}$ Creo que la traducción oficial al español habría ganado en claridad y fidelidad al original inglés si en lugar de decir "debe tener acceso a la educación" hubiera dicho "se le deberá garantizar el acceso a la educación". El texto inglés dice: "Everyone has the right to know, seek and receive information about all human rights and fundamental freedoms and should have access to human rights education and training" (art. 1.1).

${ }^{10}$ Martha Nussbaum ha defendido reiteradamente la importancia de las humanidades a la hora de formar en derechos humanos (Nussbaum, 2010). También Garrat y Piper (2011).
} 
- Aunque ya se venía insistiendo en documentos anteriores, la Declaración proclama que la educación en derechos humanos ha de abarcar todos los niveles de la educación: “art.2.2: La educación y la formación en materia de derechos humanos conciernen a todos los sectores de la sociedad, a todos los niveles de la enseñanza, incluidas la educación preescolar, primaria, secundaria y superior, teniendo en cuenta la libertad académica donde corresponda, y a todas las formas de educación, formación y aprendizaje, ya sea en el ámbito escolar, extraescolar o no escolar, tanto en el sector público como en el privado. Incluyen, entre otras cosas, la formación profesional, en particular la formación de formadores, maestros y funcionarios públicos, la educación continua, la educación popular y las actividades de información y sensibilización del público en general". Se trata de un objetivo extraordinariamente ambicioso que requiere no sólo de recursos materiales sino de una actuación coordinada entre todos los agentes implicados en la educación.

- El art. 7.4 impone un deber concreto y exigente a los Estados: “4. Los Estados y, según corresponda, las autoridades gubernamentales competentes deben garantizar la formación adecuada en derechos humanos $\mathrm{y}$, si procede, en derecho internacional humanitario y derecho penal internacional, de los funcionarios y empleados públicos, los jueces, los agentes del orden y el personal militar, así como promover la formación adecuada en derechos humanos de maestros, instructores y otros educadores y personal privado que desempeñen funciones a cuenta del Estado". Este mandato recoge lo propuesto en por la Conferencia Mundial de Derechos Humanos de 1993 y enlaza con la segunda etapa del Programa Mundial para la Educación en Derechos Humanos, que se dirige específicamente a la formación de estos colectivos. Es un acierto que se mencione a los educadores, pues tienen el deber no sólo de educar en derechos humanos sino de someterse a ellos de forma ejemplar en el desempeño de su profesión. Sin embargo, no se entiende que se omita la referencia a los profesionales sanitarios, que habían sido mencionados en la Declaración y Programa de acción de Viena.

- La Declaración concluye con un exigente mandato dirigido a los Estados: "Los Estados deben adoptar medidas adecuadas para asegurar la aplicación eficaz y el seguimiento de la presente Declaración y facilitar los recursos necesarios para ello”. Es cierto que, en estos momentos, no puede tenerse por más que una declaración de buenas intenciones. Pero, por lo menos, se reconoce que la educación en derechos humanos es lo suficientemente importante para que los Estados la garanticen y hagan efectiva mediante los recursos necesarios. 
La Asamblea General de Naciones Unidas de 2015 aprobó los Objetivos de Desarrollo Sostenible, como guía de acción de la comunidad internacional para los siguientes quince años. El cuarto de esos objetivos está monográficamente dedicado a la Educación y la Meta 7 de ese objetivo tiene directa relación con la EDH: "De aquí a 2030, asegurar que todos los alumnos adquieran los conocimientos teóricos y prácticos necesarios para promover el desarrollo sostenible, entre otras cosas mediante la educación para el desarrollo sostenible y los estilos de vida sostenibles, los derechos humanos, la igualdad de género, la promoción de una cultura de paz y no violencia, la ciudadanía mundial y la valoración de la diversidad cultural y la contribución de la cultura al desarrollo sostenible". Es cierto que no se menciona expresamente la EDH pero, como se proclama en la Declaración de Incheon de la Conferencia Mundial de la Educación de 2015, la visión de la educación en el horizonte de 2030 "se inspira en una concepción humanista de la educación y del desarrollo basada en los derechos humanos y la dignidad, la justicia social, la inclusión, la protección, la diversidad cultural, lingüística y étnica, y la responsabilidad y la rendición de cuentas compartidas” (n. 5).

\section{El Consejo de Europa (CE) y la Educación en Derechos Humanos.}

En 2010 el Comité de Ministros del CE adoptó la "Carta del Consejo de Europa sobre la educación para la ciudadanía democrática y la educación en derechos humanos". Aunque es un texto no vinculante, tiene una especial relevancia en la medida en que manifiesta el nivel el acuerdo alcanzado por los estados miembros en esta materia. También es un documento de síntesis pues el CE viene ocupándose expresamente del tema desde 1985, en que aprobó la "Recomendación sobre enseñanza y aprendizaje de los derechos humanos en la escuela" (Council of Europe, 1985). Esta primera norma ofrece todavía hoy un particular interés por el nivel de concreción de las propuestas, algo que no se ha vuelto a ver en textos análogos posteriores. Así, por ejemplo, incluye una relación de temas que deberían centrar la enseñanza en derechos humanos en la escuela $^{11}$.

\footnotetext{
11 " 3 . Knowledge to be acquired in the study of human rights

3.1. The study of human rights in schools will be approached in different ways according to the age and circumstances of the pupil and the particular situations of schools and education systems. Topics to be covered in learning about human rights could include:

i. the main categories of human rights, duties, obligations and responsibilities;

ii. the various forms of injustice, inequality and discrimination, including sexism and racism;

iii. people, movements and key events, both successes and failures, in the historical and continuing struggle for human rights;
} 
Entre 1985 y la Carta de 2010 el CE aprobó tres documentos fundamentales en esta materia. En 1997 la Asamblea General aprobó la "Recomendación sobre educación en derechos humanos" (Council of Europe, 1998). En ella se consolida una característica que define la política del CE en esta materia: hablar de derechos humanos es, sobre todo, hablar de lucha contra el racismo, la xenofobia y la intolerancia ${ }^{12}$. Si bien es cierto que estos comportamientos son lo contrario a los derechos humanos, no se puede desconocer que el alcance de los derechos humanos es mucho más amplio. Es interesante hacer notar que una organización regional semejante, como la Organización de Estados Americanos, pone en cambio el acento en el derecho a la paz y la lucha contra la violencia y en los derechos económicos, sociales y culturales.

Con ocasión de la celebración del 50 aniversario del CE en 1999, el Comité de Ministros adoptó una "Declaración sobre la educación para la ciudadanía democrática, basada en los derechos y responsabilidades de los ciudadanos". A partir de entonces la educación para la ciudadanía democrática y la educación para los derechos humanos irán de la mano, como se pone expresamente de manifiesto en la Carta de 2010.

En 2002 se aprueba la "Recomendación sobre educación para la ciudadanía democrática" (Council of Europe, 2002), que constituye el antecedente para el Año Europeo de la Ciudadanía a través de la Educación, que tuvo lugar en 2005. Desde entonces, y en paralelo con los esfuerzos llevados a cabo por Naciones Unidas en este terreno, el $\mathrm{CE}$ ha venido trabajando intensamente tanto para elaborar materiales accesibles a todos los agentes implicados, como para redactar y aprobar un documento de síntesis y consenso, como fue la Carta de 2010. En ella encontramos muchos elementos comunes a otros textos precedentes, pero cabe destacar tres aspectos originales:

- En el apartado dedicado a definiciones se distingue entre los tres niveles en los que se desarrolla la educación y en los que se tiene igualmente que intervenir a la hora de educar en derechos humanos. Son la educación formal, integrada por la enseñanza preescolar, primaria, secundaria y superior; la educación no formal, que es la que se lleva a cabo fuera de las aulas pero con fines de formación concretos; y la educación

iv. the main international declarations and conventions on human rights, such as the Universal Declaration of Human Rights and the Convention for the Protection of Human Rights and Fundamental Freedoms"; Council of Europe, Recommendation (85) 7 of the Committee of ministers to Member States on teaching and learning about human rights in schools, cit.

12 "11. The Assembly therefore recommends that the Committee of Ministers call on member states: $(\ldots)$ iv. to encourage politicians and the media to commit themselves publicly to the protection of human rights, inter alia, by checking and vigorously dismissing racist, xenophobic or intolerant declarations" (Council of Europe, 1998). 
informal, que abarca el resto de ambientes en los que las gentes desarrollan su vida (Council of Europe, 2010).

- La Declaración deja claro que el objetivo de la educación en derechos humanos no es sólo proveer de unos conocimientos sino reforzar la capacidad de acción de los ciudadanos en el seno de la sociedad ${ }^{13}$. Por un lado, parece razonable que sea así, pues la educación para la ciudadanía democrática y los derechos humanos no pretende solo una instrucción teórica sino una formación que se manifieste en acciones responsables. Pero inevitablemente se plantean dos problemas.

El primero consiste en los graves desacuerdos que se puedan plantear a la hora de actuar, porque lo que para unos será la defensa de un derecho humano para otros podrá ser un atentado contra ellos (Bellver, 2009: 31-50). Aunque la relación de situaciones de potencial conflicto sería inacabable, me limitaré a poner un ejemplo. ¿Debe presentarse la desobediencia civil como un ejemplo de ciudadanía o, más bien, de voluntad de resistirse al Estado de Derecho? Calificar ahora a Thoreau como ciudadano ejemplar es sencillo, pero ¿debemos pensar que todo el que se encadena a las puertas de un edificio público para protestar por una medida que considera gravemente injusta es un referente en la lucha por los derechos humanos?

El segundo consiste en determinar el alcance de la educación y concretamente de la enseñanza en la escuela. La capacidad de acción se refuerza de forma más efectiva trabajando en la formación afectiva y emocional. Pero, ¿es competencia de la escuela intervenir ahí? ¿No son ámbitos que corresponden primaria y casi fundamentalmente a los padres? Y aunque estimemos lícita la actuación de la escuela, ¿no es excesivo el riesgo de que esas intervenciones se conviertan en ejercicios de manipulación?

- La Declaración insiste en la necesidad de promover la investigación en esta área. Pero ese impulso a la investigación no incluye lo que debería centrar los principales esfuerzos: las dificultades para determinar los contenidos tanto de la educación para la ciudadanía democrática como de la educación en derechos humanos ${ }^{14}$. Es cierto que

13 " $5 . \mathrm{g}$. One of the fundamental goals of all education for democratic citizenship and human rights education is not just equipping learners with knowledge, understanding and skills, but also empowering them with the readiness to take action in society in the defence and promotion of human rights, democracy and the rule of law" (Council of Europe, 2010).

14 "12. Research: Member states should initiate and promote research on education for democratic citizenship and human rights education to take stock of the current situation in the area and to provide stakeholders including policy makers, educational institutions, school leaders, teachers, learners, nongovernmental organisations and youth organisations with comparative information to help them measure and increase their effectiveness and efficiency and improve their practices. This research could include, inter alia, research on curricula, innovative practices, teaching methods and development of evaluation systems, including evaluation criteria and indicators. Member states should share the results of their research with other member states and stakeholders where appropriate" (Council of Europe, 2010). 
existen áreas en las que existe un acuerdo casi universal, pero en muchas otras el choque de posiciones es frontal porque, como ya he dicho, existen no pocos comportamientos que unos califican como atentados contra los derechos humanos y otros como ejercicio legítimo de los mismos.

\section{Sobre la implementación de la educación en derechos humanos en la Universidad.}

España tiene la obligación jurídica y moral de desarrollar la EDH, no solo porque ha ratificado instrumentos legales internacionales que le obligan a ello, sino también porque ha participado y apoyado declaraciones y planes internacionales dirigidos a ese objetivo (Villán Durán, 2006: 41-66). Para cumplir con esta obligación, el Plan de Derechos Humanos (Gobierno de España, 2008) que aprobó el Gobierno de España en 2008 incluía una serie de medidas específicamente encaminadas a la formación en derechos humanos tanto de las fuerzas y cuerpos de seguridad del Estado (medida 103) como de los profesores. Concretamente se proponía que los Másteres de formación del profesorado, tanto de enseñanza primaria como secundaria, tuvieran formación específica en derechos humanos (medidas 118 y 119). También se incluía una medida dirigida a colaborar con los medios de comunicación en la sensibilización y promoción de los derechos humanos (medida 126) y, por supuesto, la incorporación en el curriculum escolar de una materia de Educación para la ciudadanía y derechos humanos.

Desafortunadamente, se ha avanzado poco en la consecución de esos objetivos. Los planes de estudio de los programas de postgrado son competencia de las respectivas universidades y el gobierno tiene un margen limitado de iniciativa para que introduzcan esta formación. Por ello, en el Informe de evaluación del Plan de Derechos Humanos de 2008 se reconoce que lo único que se ha hecho hasta ese momento en relación con las medidas 118 y 119 es poner en marcha un Grupo de trabajo tanto en el Consejo de Universidades como en la Conferencia General de Política Universitaria (Ministerio de la Presidencia, 2012: 87). No parece que ese grupo de trabajo haya conseguido resultados reseñables hasta la fecha, pues los Másteres que habilitan para impartir docencia en el sistema educativo español siguen sin contar con la materia de derechos humanos, salvo en alguno de ellos de forma accidental.

La medida 117, sobre la implantación de la asignatura de Educación para la Ciudadanía y los Derechos Humanos, que se daba por cumplida en el Informe de 2012, sufrió un 
revés en 2016, cuando el gobierno del Partido Popular decidió suprimirla. El hecho de que la controversia haya acompañado la puesta en marcha de esta materia desde sus inicios (Ruano, 2008: 251-326) no es razón suficiente, a mi entender, para suprimirla sino, más bien, para replantearla en unos términos que resulte apreciada por todos y adecuada para formar, que no adoctrinar, a los jóvenes en su condición de ciudadanos.

En diciembre de 2018 el Gobierno anunció la elaboración de un segundo Plan de Derechos Humanos que no pudo culminarse por el fin anticipado de la legislatura. Sería deseable que la nueva acometiera la aprobación de ese segundo Plan de Derechos Humanos, y que en él se contemplen medidas en relación con la EDH que se lleven efectivamente a cabo y cuya efectividad pueda ser evaluada.

En sintonía con las sucesivas etapas del Programa sobre EDH de Naciones Unidas, España ha prestado alguna atención a la formación de las fuerzas y cuerpos de seguridad del Estado, a la incorporación de esta materia en la enseñanza primaria y secundaria, y a la colaboración con los medios de comunicación. Pero, en mi opinión, el próximo Plan Nacional de Derechos Humanos debería ir más allá, impulsando algunas actuaciones estratégicas en el ámbito de la Universidad, si queremos tomarnos en serio la EDH.

La primera estaba ya apuntada en el Plan de Derechos Humanos de 2008, aunque no ha sido objeto de avances significativos. Me refiero a la mencionada incorporación de la asignatura de Derechos Humanos en todos los Másteres de Formación del Profesorado. En la medida en que se dé este paso adelante se logrará una mejora en la formación de los futuros profesores y el sistema educativo en su conjunto irá tomando conciencia del carácter trasversal que ha de tener la EDH en el currículum del estudiante.

La segunda consiste en implantar una materia de derechos humanos en todos los grados universitarios. En la actualidad esta formación suele impartirse en grados como Derecho, Ciencia Política o Trabajo Social. Otros grados incluyen alguna referencia a los derechos humanos en la medida en que pueden resultar afectados por las profesiones para las que preparan a los estudiantes. Es el caso de los grados relacionados con Ciencias de la Salud cuando tratan de los aspectos legales de las profesiones sanitarias $\mathrm{y}$, entre ellos, de los derechos del usuario del sistema sanitario. Pero hay una mayoría de grados que no incluyen referencia alguna a los derechos humanos en sus planes de estudio. ¿Es razonable que la inmensa mayoría de los universitarios llegue a incorporarse al mundo del trabajo sin haber reflexionado sobre la importancia de los derechos humanos en su vida diaria, en su desempeño profesional, en la organización política de su país y del mundo? 
Aun en el caso de que se replanteara la materia de Educación para la ciudadanía y los derechos humanos en unas condiciones adecuadas en el marco de la educación primaria y secundaria, como sería deseable que sucediera, tiene pleno sentido que todo estudiante universitario tenga también la oportunidad de recibir formación de nivel superior sobre los derechos humanos. Idealmente la formación en esta materia se podría plantear mediante dos tipos de acciones. De una parte, incorporando una asignatura específica en todos los grados, al menos con carácter voluntario; de otra, animando a que todas las materias incorporaran una perspectiva de Derechos Humanos.

La tercera, que se deriva directamente de la anterior, consistiría en formar adecuadamente a quienes fueran a impartir esta materia tanto en los Másteres de educación primaria y secundaria como en los grados universitarios. Es cierto que existe en España una nutrida oferta de Másteres en Derechos Humanos. Pero convendría que algunos de ellos desarrollaran una orientación pedagógica, que prestara atención ya no solo a los contenidos sino a las metodologías más idóneas para formar en esta materia. $\mathrm{Y}$ contando con que no todos los profesores que fueran a impartir esta docencia dispusieran del tiempo para realizar un máster, quizá se podría definir un módulo formativo de carácter obligatorio centrado en las competencias específicas exigibles en el campo de la EDH a nivel universitario. Ciertamente no es fácil acordar cuáles han de ser porque la $\mathrm{EDH}$ se puede plantear como una formación más teórica o más práctica, más centrada en transmitir conocimientos o en cultivar actitudes (Enríquez, 2014: 262). Aunque cada uno de estos dos planteamientos ofrece ventajas y problemas, no es el momento de tratarlos en profundidad. Quizá mediante un método de reflexión racional, crítico, totalizador y comprometido, propio de la Filosofía del Derecho (Solanes, 2018) se podría alcanzar lo más provechoso tanto de un enfoque teórico como práctico de la EDH. En todo caso, la literatura (Talavera, 2006) y el cine (Ruiz Sanz, 2014) (De Lucas, 2014) brindan, por el carácter narrativo que comparten con el Derecho, una magnífica aproximación al estudio de los derechos humanos en todos los niveles de la educación.

La cuarta y última actuación tiene un carácter preventivo y consiste en evitar que esta formación incurra en cuatro riesgos que anularían por completo su eficacia: la manipulación "inadvertida", la dispersión, la irrelevancia y la confusión. El riesgo de manipulación "inadvertida” se da en cualquier materia que tenga un carácter valorativo. Es obvio que cualquier iniciativa de formación en esas materias se realiza desde una moral particular. El desafío está en conseguir que el profesor, identificando ese 
presupuesto ineludible desde el que desempeña su trabajo, evite imponer su cosmovisión particular como si de un presupuesto incuestionable se tratara. No estoy hablando de burdo adoctrinamiento o intencionada manipulación ${ }^{15}$, sino de algo más sutil y menos grave, pero también negativo para el logro de una formación crítica y rigurosa. En segundo lugar, la dispersión es fácil que comparezca cuando existen tantas iniciativas que tratan de cuestiones relacionadas entre sí. Tanto los movimientos sociales como las principales organizaciones intergubernamentales han impulsado propuestas educativas centradas en la paz y la no violencia, la igualdad y la diversidad, el desarrollo sostenible, el medio ambiente, la interculturalidad, la salud, los derechos humanos, la ciudadanía, etc ${ }^{16}$. Teniendo cada una su especificidad, entiendo que la perspectiva de los derechos humanos ${ }^{17}$ puede servir de paraguas que recoja los aspectos esenciales contenidos en todas ellas ${ }^{18}$ y evitar una dispersión de esfuerzos que podría reducir su eficacia. En tercer lugar, la irrelevancia es un riesgo que acompaña a materias que carecen de un alcance utilitario inmediato y que fácilmente se pueden identificar con propuestas idealistas e ingenuas. Por último, si en EDH no se incide suficientemente en las bases ampliamente compartidas y, por el contrario, se subrayan desproporcionadamente los muchos aspectos controvertidos que entraña (Ballesteros, 1982: 239-242), es probable que se siembre la confusión y, con ella, el desencanto o simplemente el desinterés por estas cuestiones que nos interpelan a todos.

\footnotetext{
${ }^{15}$ Dice Condorcet que en todas las materias cuyas verdades no se puedan sustentar en prueba, "como influyen mucho más en la felicidad de los hombres, es mucho más importante que el poder público no dicte la doctrina común del momento como verdades eternas, por medio que haga de la instrucción un medio de consagrar los prejuicios que le son útiles, y un instrumento de poder de lo que debe ser la barrera más segura contra todo poder injusto" (Condorcet, 2001: 105).

${ }^{16}$ La Declaración de Incheon hace referencia a algunas de las propuestas de formación en valores que se han impulsado tanto desde las ONG como desde las organizaciones intergubernamentales: "la educación de calidad propicia el desarrollo de las competencias, los valores y las actitudes que permiten a los ciudadanos llevar vidas saludables y plenas, tomar decisiones con conocimiento de causa y responder a los desafíos locales y mundiales mediante la educación para el desarrollo sostenible (ESD) y la educación para la ciudadanía mundial (ECM). A este respecto, apoyamos firmemente la aplicación del Programa de acción mundial de EDS presentado en la Conferencia Mundial de la UNESCO sobre EDS que se celebró en Aichi-Nagoya en 2014. Además, destacamos la importancia de la educación y la formación en materia de derechos humanos para lograr la agenda para el desarrollo sostenible después de 2015" (UNESCO, 2015)

17 "La educación básica y especializada y la transferencia de conocimientos sobre los derechos humanos, un mandato de la ONU y la Unesco, forman parte de lo que nos gusta llamar cultura de los derechos, que muchos consideramos la base imprescindible para la tarea política por excelencia, esa permanente paideia que es la formación crítica de la ciudadanía" (De Lucas, 2019).

${ }^{18}$ La propia Declaración de Incheon apunta en esta dirección, al afirmar que la visión de la educación para el horizonte de 2030 "se inspira en una concepción humanista de la educación y del desarrollo basada en los derechos humanos y la dignidad, la justicia social, la inclusión, la protección, la diversidad cultural, lingüística y étnica, y la responsabilidad y la rendición de cuentas compartidas. Reafirmamos que la educación es un bien público, un derecho humano fundamental y la base para garantizar la realización de otros derechos"; ibídem, n. 5.
} 


\section{Conclusiones}

Del repaso a los instrumentos internacionales que se ocupan de la EDH, y de la situación de la EDH en España, podemos extraer algunas conclusiones:

1.- Si bien la idea de la EDH está presente en la misma DUDH, es a partir de 1993 cuando el tema se convierte en objeto de creciente interés por las organizaciones intergubernamentales que tratan de los derechos humanos tanto a nivel global como regional. A partir de 2005, coincidiendo con el fin del Decenio de Naciones Unidas sobre la Educación en Derechos Humanos y el inicio del Programa Mundial de Educación en Derechos Humanos, se produce una eclosión de instrumentos jurídicos dedicados a la cuestión que no ha cesado hasta el presente.

2.- A pesar del interés manifestado por Naciones Unidas y las demás organizaciones intergubernamentales para que los Estados promuevan efectivamente la EDH los resultados hasta el momento son modestos. Quizá no solo se deba a la necesidad de incrementar los recursos económicos destinados a este objetivo, sino también a la dificultad de plantear un programa sólido, coherente y atractivo, con una metodología adecuada y una inserción oportuna en los sistemas de educación formal. Para lograrlo, sería deseable que se promoviera la investigación en los problemas sustantivos que plantea EDH (Garrat y Piper, 2011: 71-84).

3.- Aunque sobre todo se insiste en incorporar la EDH en la educación primaria y secundaria, es imprescindible implicar también a la Universidad en este proyecto por dos motivos. Primero, porque le compete la formación de los profesores que vayan a impartir EDH en los colegios e institutos. Y segundo, porque parece razonable que todo estudiante universitario acceda a una EDH que le permita desarrollar la reflexión crítica y el compromiso cívico sobre los derechos humanos en el mundo y en sus entornos más próximos. En ese sentido, se deberían adoptar cuatro actuaciones: incorporar la asignatura de Derechos Humanos en todos los Másteres de Formación del Profesorado; implantar una materia de derechos humanos en todos los grados universitarios; formar adecuadamente a quienes fueran a impartir esta materia tanto en los Másteres de educación primaria y secundaria como en los grados universitarios; y evitar que esta formación incurra en los riesgos que anularían por completo su eficacia: la manipulación “inadvertida”, la dispersión, la irrelevancia y la confusión. 


\section{Referencias bibliográficas}

Acebal Monfort, L. (2006) "Programa Mundial para la Educación en Derechos Humanos y Plan de Acción 2005-2007”, en: AAVV, Educación en Derechos Humanos, Programa de Cooperación en Derechos Humanos, México, México-Comisión Europea, pp. 41-66.

Alto Comisionado de las Naciones Unidas para los Derechos Humanos. (2005). Proyecto revisado del plan de acción para la primera etapa (2005-2007) del Programa Mundial para la educación en derechos humanos, A/59/525/Rev.1, 2 de marzo de 2005. Alto Comisionado de las Naciones Unidas para los Derechos Humanos. (2014). Plan de acción para la tercera etapa (2015-2019) del Programa Mundial para la educación en derechos humanos, A/HRC/27/28, 4 de agosto de 2014.

Alto Comisionado de Naciones Unidas para los Derechos Humanos. (2015) Evaluación de la aplicación de la segunda etapa del Programa Mundial para la Educación en Derechos Humanos, A/HRC/30/24, 16 de julio de 2015.

Asamblea General de Naciones Unidas. (2012). Resolución 66/137 sobre la Declaración de Naciones Unidas sobre la educación y formación en materia de derechos humanos, A/RES/66/137, 16 de febrero de 2012.

Ballesteros, J. (1982). "Derechos humanos: ontología versus reduccionismos”, Persona y Derecho, $\mathrm{n}^{\circ}$ 9, pp. 239-242.

Bellver Capella, V. (2009). "Reflexiones sobre el lenguaje de los derechos humanos en el LX aniversario", Scio, n $^{\circ}$. 4, pp. 31-50.

Bellver Capella, V. (2013). "La educación de acuerdo con los derechos humanos", en: Narciso Martínez, Ana María Marcos, Rafael Junquera (coords.), Derechos humanos: problemas actuales. Estudios en homenaje al prof. Benito de Castro Cid, Madrid, Editorial Universitas, vol. I, pp. 269-287.

Comisión de Derechos Humanos, (2004a). Decenio de las Naciones Unidas para la Educación en la Esfera de los Derechos Humanos, (1995-2004): Informe sobre los logros y los fallos registrados en el Decenio y sobre las futuras actividades de las Naciones Unidas en esta esfera; E/CN.4/2004/93; 26 de febrero de 2004.

Comisión de Derechos Humanos. (2004b). Medidas complementarias del Decenio de las Naciones Unidas para la educación en la esfera de los derechos humanos (19952004), incluida la proclamación del Programa Mundial para la Educación en Derechos Humanos, E/CN.4/2005/98, 30 de diciembre de 2004. 
Condorcet, N. (2001). Cinco memorias sobre la instrucción pública y otros escritos, Madrid, Morata.

Consejo de Derechos Humanos. (2009). Resolución 12/4 sobre el Programa Mundial para la Educación en Derechos Humanos, 4 de diciembre de 2009, A/HRC/RES/12/4.

Consejo de Derechos Humanos. (2010). Proyecto de plan de acción para la segunda etapa (2010-2014) del Programa Mundial para la educación en derechos humanos, A/HRC/15/28, 27 de julio de 2010.

Council of Europe. (1985). Recommendation (85) 7 of the Committee of ministers to Member States on teaching and learning about human rights in schools, 14 de mayo de 1985.

Council of Europe. (1998). Recommendation 1346 (1997) on human rights education, 3 de octubre de 1998.

Council of Europe. (2002). Recommendation (2002)12 of the Committee of Ministers to member states on education for democratic citizenship, 16 de octubre de 2002.

Council of Europe. (2010). Charter on Education for Democratic Citizenship and Human Rights Education, 11 de mayo de 2010.

De Lucas, J. (2014). "Sobre Cine, Literatura y Derecho: una aproximación”, Revista de educación y derecho, 9. http://dx.doi.org/10.1344/REYD2014.9.13272 (acceso el 22 de febrero de 2019).

De Lucas, J. (2019). "La cultura de los derechos", El País, 22 de febrero de 2019, https://elpais.com/elpais/2019/02/21/opinion/1550767852_159517.html (acceso el 22 de febrero de 2019).

Enríquez, J. M. et alt. (2014). Educación plena en derechos humanos, Madrid, Trotta, pp. 262 y ss.

Garrat, D. y Piper, H. (2011). "Citizenship Education and Philosophical Enquiry: Putting Thinking Back into Practice", Education, Citizenship and Social Justice, 7, pp. $71-84$.

Gobierno de España. (2008). Plan de Derechos Humanos, aprobado por Acuerdo del Consejo de Ministros de 12 de diciembre de 2008.

Guttman, A. (2001). La educación democrática. Una teoría política de la educación,. Barcelona: Paidós.

Ministerio de la Presidencia, Plan de Derechos Humanos. Informe de Evaluación, Gobierno de España, noviembre de 2012. 
Naciones Unidas, (1994). Plan de Acción de las Naciones Unidas para la Educación en la Esfera de los Derechos Humanos, 1995-2004, n. 10; A/49/261-E/1994/110/Add.1, anexo;

https://digitallibrary.un.org/record/223443/files/A_51_506-ES.pdf (acceso el 20 de febrero de 2019).

Naciones Unidas. (2005). Programa Mundial para la educación en derechos humanos, A/RES/59/113, 17 de febrero de 2005.

Naciones Unidas. (2010). Evaluación final de la aplicación de la primera etapa del Programa Mundial para la educación en derechos humanos, A/65/322, 24 de agosto de 2010 (resumen).

Nussbaum, M. (2010). Sin fines de lucro. Por qué la democracia necesita de las humanidades, Madrid, Katz.

Ruano, L. (2008). “Objeción de conciencia a la Educación para la Ciudadanía”, Revista General de Derecho Canónico y Derecho Eclesiástico del Estado, 17, pp. 251-326.

Ruiz Sanz, M. (2014). "Instruir en Derecho y Cine: una apuesta entre elecciones y pasiones", Revista de educación y derecho, 9, http://dx.doi.org/10.1344/REYD2014.9.13049 (acceso el 22 de febrero de 2019).

Solanes, Á. (2018). "La importancia de la filosofía del derecho en los planes de estudio jurídicos dentro del EEES", Revista de educación y derecho, $\mathrm{n}^{\circ} 17$.

Talavera, P. (2006). Derecho y literatura, Granada, Comares.

UNESCO. (1995). Conferencia Internacional de Educación, Declaración y Plan de Acción Integrado sobre la Educación para la Paz, los Derechos Humanos y la Democracia, ratificada por la 28 a Asamblea General de la UNESCO, noviembre 1995.

UNESCO. (2015). Declaración de Incheon y Marco de Acción para la realización del Objetivo de Desarrollo Sostenible $4, \quad$ n. 9 , https://unesdoc.unesco.org/ark:/48223/pf0000245656_spa (acceso el 20 de febrero de 2019).

UNESCO. (2017). Informe de síntesis sobre la Aplicación por los Estados Miembros de la Recomendación de 1974 sobre la Educación para la Comprensión, la Cooperación y la Paz Internacionales y la Educación relativa a los derechos humanos y a las libertades públicas, 39 C/25, París 30 de octubre de 2017; https://unesdoc.unesco.org/ark:/48223/pf0000259734_spa (acceso 22 de febrero de 2019). 
VICENTE BELLVER CAPELLA. Educar en Derechos Humanos: orientaciones del Derecho internacional e

Villán Durán, C. (2006). “Las obligaciones de los Estados en materia de Educación en

Derechos Humanos"; en: AAVV, Educación en Derechos Humanos, Programa de

Cooperación en Derechos Humanos, México, México-Comisión Europea, pp. 41-66. 